\title{
The relationship between music and language
}

\section{Lutz Jäncke*}

Division Neuropsychology, Institute of Psychology, University of Zurich, Zurich, Switzerland

${ }^{*}$ Correspondence: I.jaencke@psychologie.uzh.ch

Traditionally, music and language have been treated as different psychological faculties. This duality is reflected in older theories about the lateralization of speech and music in that speech functions were thought to be localized in the left and music functions in the right-hemisphere of the brain. For example, the landmark paper of Bever and Chiarello (1974) emphasized the different roles of both hemispheres in processing music and language information, with the left hemisphere considered more specialized for propositional, analytic, and serial processing and the right-hemisphere more specialized for appositional, holistic, and synthetic relations. This view has been challenged in recent years mainly because of the advent of modern brain imaging techniques and the improvement in neurophysiological measures to investigate brain functions. Using these innovative approaches, an entirely new view on the neural and psychological underpinnings of music and speech has evolved. The findings of these more recent studies show that music and speech functions have many aspects in common and that several neural modules are similarly involved in speech and music (Tallal and Gaab, 2006). There is also emerging evidence that speech functions can benefit from music functions and vice versa. This field of research has accumulated a lot of new information and it is therefore timely to bring together the work of those researchers who have been most visible, productive, and inspiring in this field.

This special issue comprises a collection of 20 review and research papers that focus on the specific relationship between music and language. Of these 20 papers 12 are research papers that report entirely new findings supporting the close relationship between music and language functions. Two papers report findings demonstrating that phonological awareness, which is pivotal for reading and writing skills, is closely related to pitch awareness and musical expertise (Dege and Schwarzer, 2011; Loui et al., 2011). Dege and colleagues even show that pre-schoolers can benefit from a program of musical training to increase their phonological awareness.

Three research papers focus on the relationship between tonal language expertise and musical pitch perception skills and on whether pitch-processing deficits might influence tonal language perception. Giuliano et al. (2011) demonstrated Mandarin speakers are highly sensitive to small pitch changes and interval distances, a sensitivity that was absent in the control group. Using ERPs obtained during the pitch and interval perception tasks, their study reveals earlier ERP responses in Mandarin speakers compared with controls to these pitch changes relative to no-change trials. In their elegant paper, Peretz et al. (2011) report that native speakers of a tone language, in which pitch contributes to word meaning, are impaired in the discrimination of falling pitches in tone sequences as compared with speakers of a non-tone language. Taken together, these two studies illustrate the cross-domain influence of language experience on the perception of pitch, suggesting that the native use of tonal pitch contours in language leads to a general enhancement in the acuity of pitch representations. Tillmann et al. (2011) examined whether subjects suffering from congenital amusia also demonstrate impairments of pitch-processing in speech, specifically the pitch changes used to contrast lexical tones in tonal languages. Their study revealed that the performance of congenital amusics was inferior to that of controls for all materials including the Mandarin language, this therefore suggesting a domain-general pitch-processing deficit.

Five research papers sought to examine interactions either between musical expertise and language functions or whether an interaction between musical and language functions is beneficial for phonetic perception. Ott et al. (2011) demonstrate that professional musicians process unvoiced stimuli (irrespective of whether these stimuli are speech or non-speech stimuli) differently than controls, this suggesting that early phonetic processing is differently organized depending on musical expertise. Strait and Kraus (2011) report perceptual advantages in musicians for hearing and neural encoding of speech in background noise. They also argue that musicians possess a neural proficiency for selectively engaging and sustaining auditory attention to language and that music thus represents a potential benefit for auditory training. Gordon et al. (2011) examined the interaction between linguistic stress and musical meter and established that alignment of linguistic stress and musical meter in song enhances musical beat tracking and comprehension of lyrics. Their study thus supports the notion of a strong relationship between linguistic and musical rhythm in songs. Hoch et al. (2011) investigated the effect of a musical chord's tonal function on syntactic and semantic processing and conclude that neural and psychological resources of music and language processing strongly overlap. The fifth paper of this group (Omigie and Stewart, 2011) demonstrates that the difficulties amusic individuals have with real-world music cannot be accounted for by an inability to internalize lower-order statistical regularities but may arise from other factors. Although there are still some differences between music and speech-processing, there thus is growing evidence that speech and music processing strongly overlap.

Halwani et al. (2011) examined whether the arcuate fasciculus, a prominent white-matter tract connecting temporal and frontal brain regions, is anatomically different between singers, instrumentalists, and non-musicians. They showed that long-term vocal-motor training might lead to an increase in volume and microstructural complexity (as indexed by fractional anisotropy measures) of the arcuate fasciculus in singers. Most likely, these anatomical changes reflect the necessity in singers of strongly linking together frontal and temporal brain regions. Typically, these regions are also involved in the control of many speech functions. The beneficial impact of music on speech functions has also been demonstrated by Vines et al. (2011) in their research paper. They examined whether the melodic intonation therapy (MIT) in Broca's aphasics can be improved by simultaneously applying anodal transcranial direct current stimulation (tDCS). In fact, they showed that the combination of right-hemisphere anodal-tDCS with MIT speeded up recovery from post-stroke aphasia. 
In addition to these 12 research papers there are 8 review and opinion papers that highlight the tight link between music and language. Patel (2011) proposes the so-called OPERA hypothesis with which he explains why music is beneficial for many language functions. The acronym OPERA stands for five conditions which might drive plasticity in speech-processing networks (Overlap: anatomical overlap in the brain networks that process acoustic features used in both music and speech; Precision: music places higher demands on these shared networks than does speech; Emotion: the musical activities that engage this network elicit strong positive emotion; Repetition: the musical activities that engage this network are frequently repeated; Attention: the musical activities that engage this network are associated with focused attention). According to the OPERA hypothesis, when these conditions are met, neural plasticity drives the networks in question to function with higher precision than needed for ordinary speech communication. While Patel's paper is more an opinion paper that puts musical expertise into a broader context, the seven other reviews more or less emphasize specific aspects of the current literature on music and language. Ettlinger et al. (2011) emphasize the specific role of implicitly acquired knowledge, implicit memory, and their associated neural structures in the acquisition of linguistic or musical grammar. Milovanov and Tervaniemi (2011) underscore the beneficial influence of musical aptitude on the acquisition linguistic skills as for example in acquiring a second language. Bella et al. (2011) summarize findings of the existing literature concerning normal singing and poor-pitch singing and suggest that pitch imitation may be selectively inaccurate in the music domain without being affected in speech, thus supporting the separability of

\section{REFERENCES}

Bella, S. D., Berkowska, M., and Sowinski, J. (2011). Disorders of pitch production in tone deafness. Front. Psychol. 2:164. doi: 10.3389/fpsyg.2011.00164

Besson, M., Chobert, J., and Marie, C. (2011). Transfer of training between music and speech: common processing, attention, and memory. Front. Psychol. 2:94. doi: 10.3389/ fpsyg.2011.00094

Bever, T. G., and Chiarello, R. J. (1974). Cerebral dominance in musicians and nonmusicians. Science 185, 537-539.

Dege, F., and Schwarzer, G. (2011). The effect of a music program on phonological awareness in preschoolers. Front. Psychol. 2:124. doi: 10.3389/ fpsyg.2011.00124

Ettlinger, M., Margulis, E. H., and Wong, P.C. (2011). Implicit memory in music and language. Front. Psychol. 2:211. doi: 10.3389/fpsyg.2011.00211

Giuliano, R. J., Pfordresher, P. Q., Stanley, E. M., Narayana, S., and Wicha, N. Y. (2011). Native experience with a tone language enhances pitch discrimination and the timing of neural responses to pitch change. Front. Psychol. 2:146. doi: 10.3389/fpsyg.2011.00146

Gordon, R. L., Magne, C. L., and Large, E. W. (2011). EEG Correlates of Song Prosody: a new look at the relation- ship between linguistic and musical rhythm. Front. Psychol. 2:352. doi: 10.3389/fpsyg.2011.00352

Halwani, G. F., Loui, P., Ruber, T., and Schlaug, G. (2011). Effects of practice and experience on the arcuate fasciculus: comparing singers, instrumentalists, and non-musicians. Front. Psychol. 2:156. doi: 10.3389/fpsyg.2011.00156

Hoch, L., Poulin-Charronnat, B., and Tillmann, B. (2011). The influence of task-irrelevant music on language processing: syntactic and semantic structures. Front. Psychol. 2:112. doi: 10.3389/fpsyg.2011.00112

Koelsch, S. (2011). Toward a neural basis of music perception - a review and updated model. Front. Psychol. 2:110. doi: 10.3389/fpsyg.2011.00110

Loui, P., Kroog, K., Zuk, J., Winner, E., and Schlaug, G. (2011). Relating pitch awareness to phonemic awareness in children: implications for tone-deafness and dyslexia. Front. Psychol. 2:111. doi: 10.3389/fpsyg.2011.00111

Milovanov, R., and Tervaniemi, M. (2011). The interplay between musical and linguistic aptitudes: a review. Front. Psychol. 2:321. doi: 10.3389/ fpsyg.2011.00321

Omigie, D., and Stewart, L. (2011). Preserved statistical learning of tonal and linguistic material in congenital

mechanisms subserving pitch production in music and language. In their extensive review of the literature, Besson et al. (2011) discuss the transfer effects from music to speech by specifically focusing on the musical expertise in musicians. Shahin (2011) article reviews neurophysiological evidence supporting an influence of musical training on speech perception at the sensory level, and the question is discussed whether such transfer could facilitate speech perception in individuals with hearing loss. This review also explains the basic neurophysiological measures used in the neurophysiological studies of speech and music perception. The comprehensive review by Koelsch (2011) summarizes findings from neurophysiology and brain imaging on music and language processing and integrates these findings into a broader "neurocognitive model of music perception." Specific emphasis is placed on the comparison of musical syntax and their similarities and differences to language syntax. Schon and Francois (2011) present a review in which they focus on a series of electrophysiological studies that investigated speech segmentation and the extraction of linguistic versus musical information. They demonstrated that musical expertise facilitates the learning of both linguistic and musical structures. A further point is that electrophysiological measures are often more sensitive for identifying music-related differences than behavioral measures.

Taken together, this special issue provides a comprehensive summary of the current knowledge on the tight relationship between music and language functions. Thus, musical training may aid in the prevention, rehabilitation, and remediation of a wide range of language, listening, and learning impairments. On the other hand, this body of evidence might shed new light on how the human brain uses shared network capabilities to generate and control different functions.

amusia. Front. Psychol. 2:109. doi: 10.3389/fpsyg.2011.00109

Ott, C. G., Langer, N., Oechslin, M., Meyer, M., and Jancke, L. (2011). Processing of voiced and unvoiced acoustic stimuli in musicians. Front. Psychol. 2:195. doi: 10.3389/fpsyg.2011.00195

Patel, A. D. (2011). Why would musical training benefit the neural encoding of speech? The OPERA hypothesis. Front. Psychol. 2:142. doi: 10.3389/ fpsyg.2011.00142

Peretz, I., Nguyen, S., and Cummings, S. (2011). Tone language fluency impairs pitch discrimination. Front. Psychol. 2:145. doi: 10.3389/ fpsyg.2011.00145

Schon, D., and Francois, C. (2011). Musical expertise and statistical learning of musical and linguistic structures. Front. Psychol. 2:167. doi: 10.3389/fpsyg.2011.00167

Shahin, A. J. (2011). Neurophysiological influence of musical training on speech perception. Front. Psychol. 2:126. doi: 10.3389/ fpsyg.2011.00126

Strait, D. L., and Kraus, N. (2011). Can you hear me now? Musical training shapes functional brain networks for selective auditory attention and hearing speech in noise. Front. Psychol. 2:113. doi: 10.3389/fpsyg.2011.00113
Tallal, P., and Gaab, N. (2006). Dynamic auditory processing, musical experience and language development. Trends Neurosci. 29, 382-390.

Tillmann, B., Burnham, D., Nguyen, S., Grimault, N., Gosselin, N., and Peretz, I. (2011). Congenital amusia (or tonedeafness) interferes with pitch processing in tone languages. Front. Psychol. 2:120. doi: 10.3389/fpsyg.2011.00120

Vines, B. W., Norton, A. C., and Schlaug, G. (2011). Non-invasive brain stimulation enhances the effects of melodic intonation therapy. Front. Psychol. 2:230. doi: 10.3389/ fpsyg.2011.00230

Received: 05 April 2012; accepted:07 April 2012; published online: 27 April 2012. Citation: Jäncke L (2012) The relationship between music and language. Front. Psychology 3:123. doi: 10.3389/ fpsyg.2012.00123

This article was submitted to Frontiers in Auditory Cognitive Neuroscience, a specialty of Frontiers in Psychology. Copyright (c) 2012 Jäncke. This is an openaccess article distributed under the terms of the Creative Commons Attribution Non Commercial License, which permits non-commercial use, distribution, and reproduction in other forums, provided the original authors and source are credited. 